\title{
Robust Controller in the Structure of Lateral Control of Maneuvering Aircraft
}

\author{
Róbert Bréda*, Tobiáš Lazar*, Rudolf Andoga*, Ladislav \\ Madarász**
}

*Technical university Košice, Faculty of Aeronautics, Department of Avionics, Rampová 7, 04021 Košice, Slovak Republic, robert.breda@tuke.sk, tobias.lazar@tuke.sk, rudolf.andoga@tuke.sk

**Technical university Košice, Faculty of Electrical Engineering and Informatics Department of Cybernetics and Artificial Intelligence Letná 9, 04200 Košice, Slovak Republic, ladislav.madarasz@tuke.sk

\begin{abstract}
Efficiency in the process of aircraft control is expressed as the adaptability of the control system to the changes in the physical properties of the object. As a criterion of efficiency there exists an indicator by which the rules of selecting the best ways of solving control problems are determined. The contribution is describing the method of analyzing, synthetizing the designed parameters of a robust controller for lateral control of aircraft utilizing assisting damping automated devices (ADAD). The design of the controller parameters was done using Matlab program with the demo version of the lateral control of a maneuvering aircraft and the synthesis of the suggested controller is based on applying the $H_{\infty}$ and $\mu$ methods.
\end{abstract}

Keywords: robust controller; multiplicative uncertainty; singular values; assisting damping and automatic devices (ADAD)

\section{Introduction}

Controlling the flight of an aircraft represents a process that takes place within a closed circuit, termed as the man - machine - loop, or an automated flight control system. When controlling an aircraft, it is about evaluation of information on the real motion of the aircraft and the subsequently well - organized utilization of selected forces and momenta acting on the aircraft to ensure the required movement of its center of gravity and angular positioning of the aircraft around it.

Currently used methods of aircraft control involves those of PID regulation, robust control and progressive methods of the Fuzzy logic. In the past, PID controllers were used as autopilots, based on simple laws up to the advanced systems of 
control further developed into structures with multivariable coefficients in compliance with flight conditions, [13]. Today, advanced systems are used, which enable implementation of more complex laws of control and invariance of preset coefficients in time. When applying robust controllers, aircraft control systems make use of relatively widely used methods of $H_{\infty}$ and $\mu$ synthesis. For example, the control system of the highly maneuverable F - 16 VISTA fighter, where control in the lateral and directional axes is ensured within the internal loop by method of dynamic inversion and the external control loop is made applying the method of $\mu$ synthesis. The control system in the longitudinal axis is also divided into the internal loop, $H_{\infty}$ which is performed at of minimal order and the external loop by method of the $\mu$ synthesis, [1]. Methods of analysis and synthesis enable design of the parameters of a robust controller for lateral motion of a maneuvering aircraft, with the $H_{\infty}$ and $\mu$ synthesis methods applied in the Matlab program environment.

\section{Robust Control of Multi - Dimensional Systems}

The theory of robust control of dynamic systems is typical for modeling, analysis of the object features when controller synthesis is to be done with an incomplete and imprecise mathematical description of the process. The problem of robust stabilization can be solved by optimal control methods such as based on the minimization of norms of transfer functions of the feedback system. The notion of designing a robust controller will be understood as a procedure and resulting in the design of a controller, which ensures robust features of the closed control loop at prescribed magnitudes of uncertainties, [3, 4]. Multidimensional MIMO systems by their specific features belong to the class of hard-to-stabilize systems, using the feedback from the output variable. Problems in the design of robust controllers consist in the interactional links existing between the separate subsystems of the multidimensional system and the one of ,gains" of the multidimensional system changing between the minimum and the maximum value of the singular value of the system matrix. Multidimensional systems are substantially sensitive to the changes in the parameters than the uni - dimensional SISO systems, and therefore the design of controllers stabilizing the process or the design of robust controllers is rather difficult. Any kind of precise model of the system is only approximating the behavior of the real system. The primary role of the feedback is to eliminate the effect of uncertainty, indefiniteness, as well as to obtain the required quality in control as defined by the appropriate norms. The term „robust“ is considered for such calculation procedures, which at small changes, or errors in the input data guarantee even the proportionately smallest variations in the output results. 
Robustness is a measure to which a system is tolerant to certain limits to structured or non - structured uncertainties. Non - structured uncertainty is characterized as additional dynamics, which is not part of the model and can be of additive or even multiplicative in nature.

Real system is represented by $G(s)$, nominal system by $G_{0}(s), \Delta_{a}$ stands for additive uncertainties - unknown information on the true value and phase of perturbation and $\Delta_{m}$ for multiplicative uncertainty. For additive uncertainty it holds:

$$
G(s)=G_{0}(s)+\Delta_{a}(s)
$$

For multiplicative uncertainty at the system input it holds:

$$
G(s)=G_{0}(s)\left[I+\Delta_{m}(s)\right]
$$

For multiplicative uncertainty at the system output it holds:

$$
G(s)=\left[I+\Delta_{m}(s)\right] G_{0}(s)
$$

Structured uncertainty is represented by the uncertainty of the parameter or the set of system parameters, and it also may be of additive or multiplicative in nature. Let $l$ be a parameter of a real system, and $l_{0}$ is the nominal parameter, then for the separate uncertainty it holds:

$$
\begin{aligned}
& l=l_{0}+\Delta_{a} \\
& l=l_{0}\left(1+\Delta_{m}\right)
\end{aligned}
$$

\subsection{Method of Structured Singular Values}

The analysis of structured singular values $(\mu)$, based on the ,small gain theorem", is used to evaluate the robustness of the system. In order to find the optimal controller of the aircraft control system, it might be necessary to make use the very methods of structured singular values ( $\mu$ synthesis), [1]. The purpose of the $\mu$ synthesis is to find the controller, which minimizes the upper limit of structured singular values, see Fig. 1.

$P(s)$ is the object of control, $K(s)$ controller, $w_{0}$ input uncertainty, $w_{i}$ output multiplicative function that ensures classification of the uncertainty, $\Delta(s)$ parameter of uncertainty. 


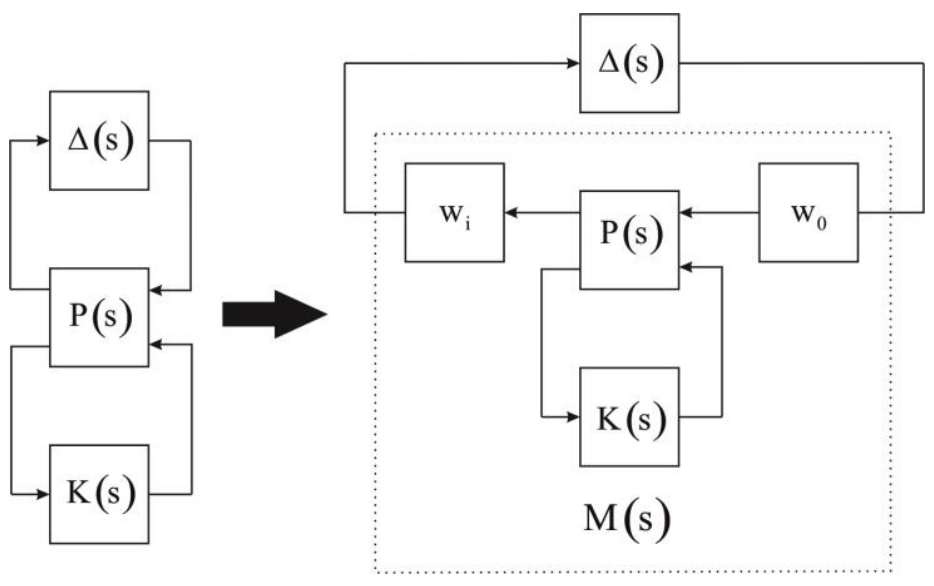

Figure 1

Block diagram of the method of structured singular values

Equivalent representation of the block of uncertainty is:

$\Delta(s)=w_{0} \Delta(s) w_{i}$, where for the norm of uncertainty it holds that $\|\Delta(s)\|_{\infty} \leq 1$

The theory of small gains guarantees that if $M(s)$ and $\Delta(s)$ are stable, then the uncertain system will remain stable, if for all the frequencies it holds that $0 \leq \omega \leq \infty$.

$\bar{\sigma}(M(j \omega) \Delta(j \omega)) \leq 1$, which can be expressed as $\|M(s) \Delta(s)\|_{\infty} \leq 1$.

The inequality can be written as $\|M(s) \Delta(s)\|_{\infty} \leq\|M(s)\|_{\infty}\|\Delta(s)\|_{\infty}$ because it is known that $\|\Delta(s)\|_{\infty} \leq 1$

Then, the satisfactory condition to the stability is: $\|M(s)\|_{\infty} \leq 1$

To design the controller, it is necessary to define the uncertainties, which at the design of the aircraft control system and can be divided as uncertainties in:

- $\quad$ state matrix of the aircraft linear model,

- efficiency of operating elements - actuators,

- in - flight measurement of parameters,

- neglecting tensile design of the aircraft.

Constraints such as the dynamics of operating elements, noise and filters used for processing of the input signals coming from sensors may also be included in the model. 


\subsection{The $\mathrm{H}_{\infty}$ Method}

Method $H_{\infty}$ is aimed to find the controller with $\boldsymbol{y}(t)$ inputs and $\boldsymbol{u}(t)$ outputs, i.e. the one, which eliminates transfer functions between $\boldsymbol{w}(t)$ and $z(t)$ as shown in Figure 2.

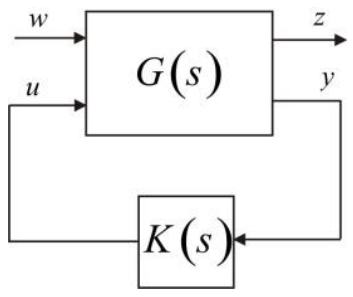

Figure 2

Block diagram of the $H_{\infty}$ method

The linear system on which the method is applied can be generally described by the equation as:

$\dot{\boldsymbol{x}}(t)=\boldsymbol{A} \boldsymbol{x}(t)+\boldsymbol{B}_{1} \boldsymbol{w}(t)+\boldsymbol{B}_{2} \boldsymbol{u}(t)$,

$\boldsymbol{z}(t)=\boldsymbol{C}_{1} \boldsymbol{x}(t)+\boldsymbol{D}_{11} \boldsymbol{w}(t)+\boldsymbol{D}_{12} \boldsymbol{u}(t)$,

$\boldsymbol{y}(t)=\boldsymbol{C}_{2} \boldsymbol{x}(t)+\boldsymbol{D}_{21} \boldsymbol{w}(t)+\boldsymbol{D}_{22} \boldsymbol{u}(t)$.

where:

$\boldsymbol{x}(t)$ is an $\mathrm{n}$ - dimensional state vector of the system, $\boldsymbol{w}(t) m_{1}$ - dimensional vector of faulty variables, $\boldsymbol{u}(t) m_{2}$ - dimensional vector of input variables, $\boldsymbol{z}(t)$ is the $p_{1}$ - dimensional vector of variables magnitudes the magnitude of which must be minimized and $\boldsymbol{y}(t)$ is the $p_{2}$ - dimensional vector of output or the measured variables. Prior to the synthesis of the controller, it is necessary to select in advance the weight functions, and also to measure the important signals, which affect the system on principle. Having extended the system by weight functions and constants, one can proceed to the design of the controller as it.

Norm $H_{\infty}$ for the real matrix $T(x)$ is defined:

$\|T\|_{\infty}=\sup _{\operatorname{Re}(s) \succ 0}\|T(s)\|=\sup _{\omega}\|T(j \omega)\|=\sup _{\omega} \bar{\sigma}[T(j \omega)]$

where:

$\bar{\sigma}[T(j \omega)]$ denotes the largest singular value of $T(j \omega)$ depending on the frequency. 
To find the controller based on the methods as above, one can make use of the „Robust Control Toolbox“ program of the Matlab environment, which enables generating a model in compliance with the equation, defining the uncertain state area, synthesis of the controller applying the $H_{\infty}$ or the $\mu$ method the one of synthesis and also reduction of the order of controller in case when it becomes apparent that the controller obtained is significantly slowing down the time of simulation [7].

\section{Non - Linear Mathematical Model of the Aircraft Motion}

Generally, a non-linear model of an aircraft consists of: model of dynamics for the determined class of aircraft in the environment, model of the power plant, model of the dynamics of operating elements, model of the atmosphere, shown in Figure 3 [2]. When modeling aircraft dynamics, the following assumptions are adopted:

- $\quad$ aircraft structure is perfectly stiff, eliminating the aero - elastic vibrations of the structure during flight,

- aircraft weight with momenta of gyro/inertia will remain constant in the process of modeling, not assuming transfer of fuel between tanks when in flight

- $\quad$ standard atmosphere as by ISA.

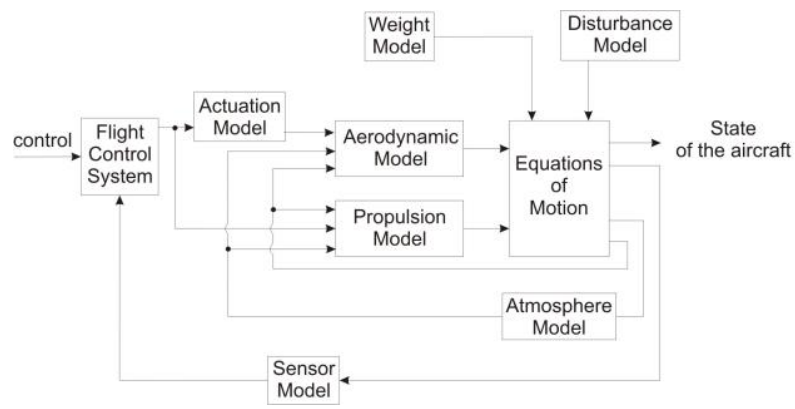

Figure 3

Block diagram of a non - linear model of an aircraft 


\subsection{Lateral Movement Equations for the Aircraft}

Environmental influence causes the movement equations of aircraft to become non - linear and their coefficients variable. Solution of such equations and their practical realization in modelling is source of substantial difficulties. In order to overcome them, the method of linearization is employed, which by selecting an operational state shows the outputs within the assumed linearization limits. It means that the process of the dynamic flight is decomposed into increments related to the referential points, which represents the starting point for determining further movement of the aircraft. Then the linear equations of motion for lateral and directional motions of the aircraft comprise the equations for force $\mathrm{Y}$, momenta L and N, [8]. For the lateral motion of the aircraft, equations of motion can be defined, which comprise three dynamical and two kinematical equations.

$$
\begin{aligned}
m \dot{v}-\dot{Y}_{v} v-\left(\dot{Y}_{p}+m W_{s}\right) p-\left(\dot{Y}_{r}-m U_{s}\right) r-m g \phi \cos \Theta_{s}-m g \psi \sin \Theta & =\dot{Y}_{\xi} \xi+\dot{Y}_{\zeta} \zeta, \\
-\dot{L}_{v} v+I_{x x} \dot{p}-\dot{L}_{p} p-I_{x z} \dot{r}-\dot{L}_{r} r & =\dot{L}_{\xi} \xi+\dot{L}_{\zeta} \zeta, \\
-\dot{N}_{v} v-I_{x z} \dot{p}-\dot{N}_{p} p+I_{z z} \dot{r}-\dot{N}_{r} r & =\dot{N}_{\xi} \xi+\dot{N}_{\zeta} \zeta, \\
\dot{\phi} & =p, \\
\dot{\psi} & =r .
\end{aligned}
$$

The lateral motion of the aircraft is described by the system of five linear differential equations of the first order with five unknown variables $v, p, r, \psi, \phi$. The equations are referenced to the body frame of the aircraft, see Fig. 4 [10]. Linear differential equations for the lateral motion of the aircraft are written as state-space equations:

$$
\begin{aligned}
\left(\begin{array}{c}
\dot{v} \\
\dot{p} \\
\dot{r} \\
\dot{\phi} \\
\dot{\psi}
\end{array}\right)=\left(\begin{array}{lllll}
y_{v} & y_{p} & y_{r} & y_{\phi} & y_{\psi} \\
l_{v} & l_{p} & l_{r} & l_{\phi} & l_{\psi} \\
n_{v} & n_{p} & n_{r} & n_{\phi} & n_{\psi} \\
0 & 1 & 0 & 0 & 0 \\
0 & 0 & 1 & 0 & 0
\end{array}\right)\left(\begin{array}{l}
v \\
p \\
r \\
\phi \\
\psi
\end{array}\right)+\left(\begin{array}{lll}
y_{\xi} & y_{\zeta} \\
l_{\xi} & l_{\zeta} \\
n_{\xi} & n_{\zeta} \\
0 & 0 \\
0 & 0
\end{array}\right)\left(\begin{array}{l}
\xi \\
\zeta
\end{array}\right) \\
\boldsymbol{y}(t)=\operatorname{Ix}(t)=\left(\begin{array}{lllll}
1 & 0 & 0 & 0 & 0 \\
0 & 1 & 0 & 0 & 0 \\
0 & 0 & 1 & 0 & 0 \\
0 & 0 & 0 & 1 & 0 \\
0 & 0 & 0 & 0 & 1
\end{array}\right)\left(\begin{array}{l}
v \\
p \\
r \\
\phi \\
\psi
\end{array}\right)
\end{aligned}
$$




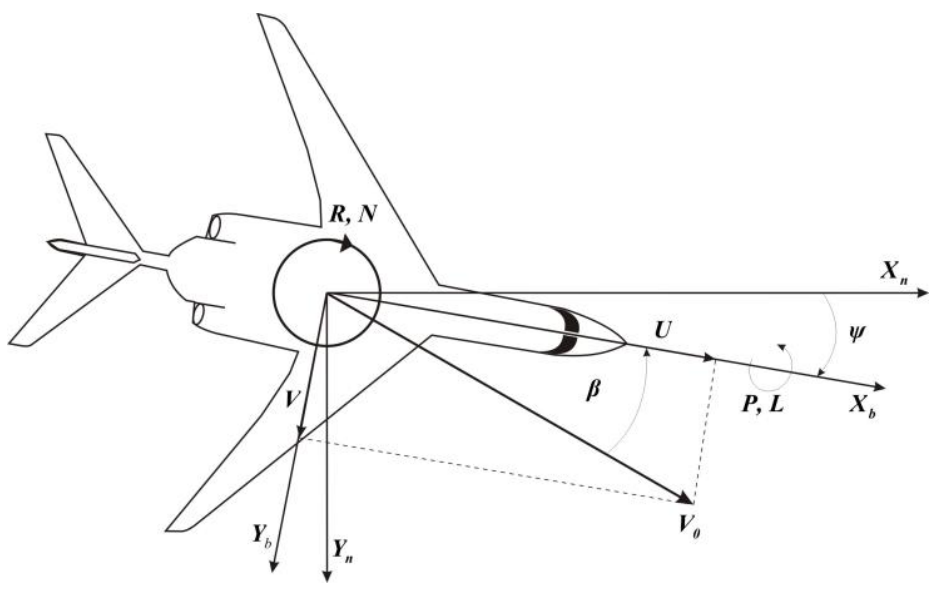

Figure 4

Lateral motion of the aircraft in coordinate systems

Dynamics of the aircraft lateral motion is characterized in damping mode - in pitching and spiral motions. Spiral motion has a great time constant, can be of mildly divergent. It is characterized by changes in the angles of banking and headings at small changes in directional movement. The damping - pitching motion is rapid and stable and describes the aircraft response to the lateral deflection of the control stick. Dutch Roll is an oscillating, poorly attenuated motion describing the aircraft response onto deflecting the pedals of directional control. The nature of the longitudinal relaxation and lateral fluctuation is unpleasant for the pilot, as it requires constant application of the means of aircraft control. Assesment of characteristic variables of oscillations and fluctiations helps to evaluate the quality of responsiveness and stability of aircraft motion which results from pilot's control actions. The frequency of oscillations and fluctuations is related to flight regime, in which the speed, altitude, acceleration multiples and the Mach number are determinant for its stability and controllability [12].

Table 1

Requirement for the value of damping by rapid movement, Dutch Roll

\begin{tabular}{|c|c|c|c|}
\hline Level & $\min \zeta_{d}$ & $\min \zeta_{d} \omega_{d}(\mathrm{rad} / \mathrm{s})$ & $\min \omega_{d}(\mathrm{rad} / \mathrm{s})$ \\
\hline \hline 1 & 0.4 & 0.4 & 1.0 \\
\hline 2 & 0.02 & 0.05 & 0.4 \\
\hline 3 & 0 & --- & 0.4 \\
\hline
\end{tabular}

Controllability of highly maneuverable aircraft is ensured by assisting damping and automatic devices (ADAD) the tasks of which is, following pilot action, to shorten, via the feedback action of the control surfaces, the time of tion from the original position to the next one without excessive motions. They all support 
efficiency of performing the manoeuver at the prescribed value for flight safety and aircraft structure. The ADAD functions are ensured via measuring aircraft position and motion at a prescribed dynamics.

\subsection{Design of a Robust Controller for Pilot - controlled Landing by the Directional Control of the Aircraft}

The presented methods of analysis and synthesis help determine the parameter of the controller illustrated in Fig. 5 with a block $K$ for the lateral motion of the aircraft. Input variables of the controller are the actual parameters of aircraft position with reference to the directional axis introduced by the pilot with the control stick handle and pedals [6]. Aircraft parameters measured by sensors:

- roll rate $p$, around the longitudinal axis $X$,

- yaw rate $r$, around the vertical axis $Z$,

- normal acceleration $\mathrm{Nz}$, along the vertical axis $\mathrm{Z}$,

- $\quad$ sideslip angle $\beta$.

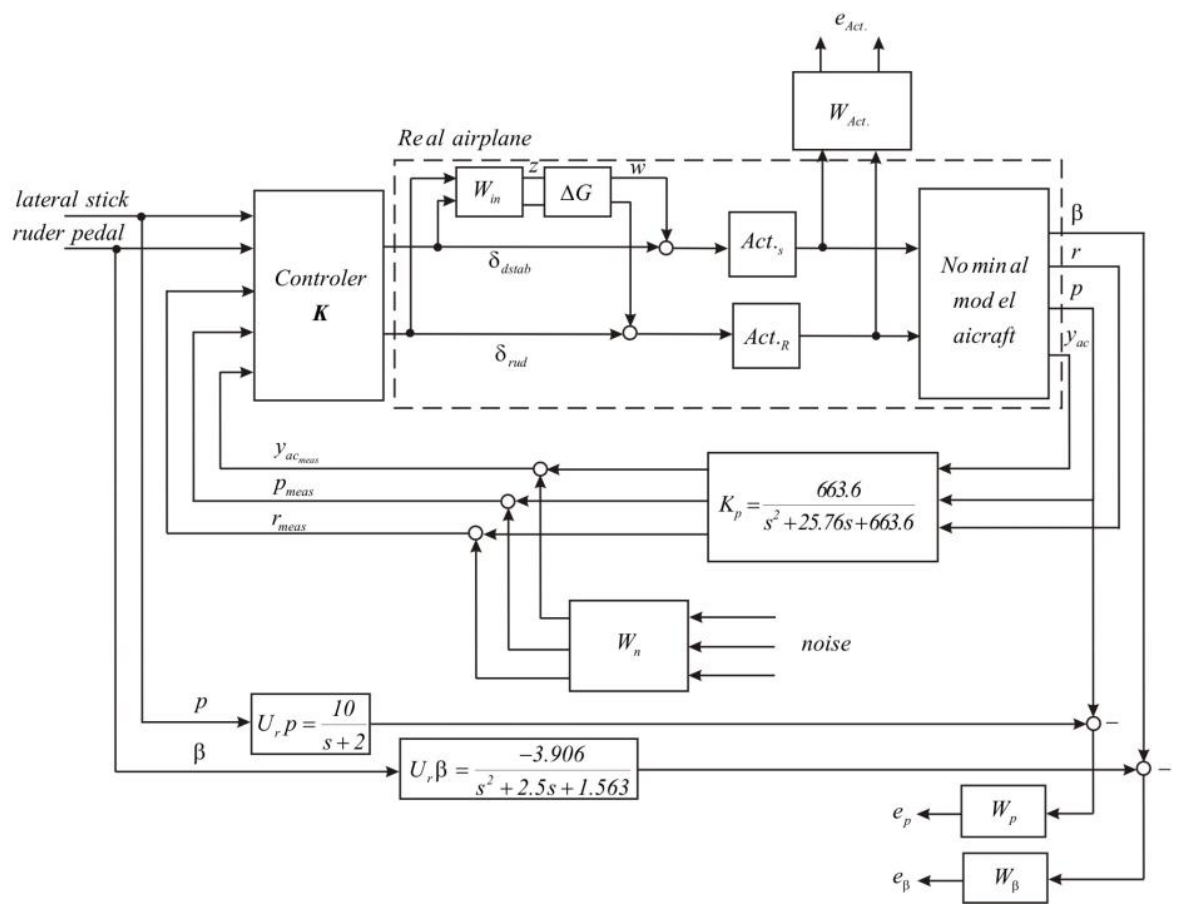

Figure 5

Integration of the robust controller in the structure of lateral movement control of the maneuvering aircraft 
Input data are standing for angle of attack $\alpha=10,5^{\circ}$ and landing speed $v=140$ knots. The aircraft control circuit features shaping filters, where the required shape of speed for roll rate $p$ caused by the pilot is adjusted by the filter with transfer function: $U_{r} p=\frac{10}{s+2}$. Quality of lateral control is increased by a filter of second order adjusted by transfer function: $U_{r} \beta=\frac{-3.906}{s^{2}+2.5 s+1.563}$.

Smooth shape of transient characteristics of time responses without excessive oscillations is precondition to qualitatively sound responses of the aircraft, its controllability, without reducing energy potential. Aircraft reactions measured by sensors are: roll rate and yaw rate $p, r$ and lateral acceleration $y a_{c}$.

Output signals of sensors are passing pass through a three - channel shaper a filter with transfer function $K_{p}=\frac{663.6}{s^{2}+25.76 s+663.6}, \quad K_{p}=K_{r}=K_{y_{-a c}}$ and sums of positive element are fed onto the input of the robust controller $K$, which makes up the assisting attenuating automatic device (ADAD). The ADAD functions are ensured via measuring the position of aircraft with a prescribed dynamics.

Servo elements of the ADAD are the rudder actuators with prescribed data:

- stabilizer deflection: $\pm 20^{\circ}$ degrees, motion speed: $\pm 90^{\circ} / \mathrm{sec}$,

- rudder deflection: $\pm 30^{\circ}$ degrees, motion speed: $\pm 125^{\circ} / \mathrm{sec}$.

Measurement of aircraft movement around the $X, Z$ axes and in the direction of $Z$ axis is performed by sensors of angular velocities/gyro/ and accelerations with prescribed features. Noisy signals of normal accelerations and angular velocities are separated from the useful by filters, which also ensure shaping for further use. Input frequency measured by the sensor at aircraft turn is $f=12,5 \mathrm{~Hz}$, sensor dynamics of $f=25 \mathrm{~Hz}$, damping $\zeta=0,7$. Flight of the aircraft in the direction is influence by natural fluctuation at a frequency of $f=4,1 \mathrm{~Hz}$.

\subsection{Determining the Weight Functions of the Controller}

Algorithms of robust control minimize action of the feed-back loop by reducing its gain by the frequency of aircraft fluctuation $H_{\infty_{\text {nominal }}}$. To determine the true frequency of fluctuation, weight functions are used, when the pulses to obtain weight responses on the aircraft are generated by interceptors, operated by pilot's control stick handle until the aircraft reaction is achieved in the form of weight function $p, r, y_{a_{c}}$, the signals of which enter the joint filter - the shaper. The numeric values of the filters and models have been taken from [7]. 
Additive noises are eliminated by another joint filter-shaper, which is synthetized by setting up a diagonal matrix of the known values of active elements (interceptor, rudder, angular velocities, positions) [7]:

$$
\text { Wact }=\operatorname{diag}([1 / 90,1 / 20,1 / 125,1 / 30])=\operatorname{diag}([0.0111,0.5,0.008,0.0333])
$$

Of the matrix mentioned, the transfer functions are formulated, which are equations of shaping filters of the upper - values marked as $W n$ in Fig. 6, which combines filters $W n_{1}=0.025, \quad W n_{2}=\frac{0.0125 s+0.0125}{s+100}, \quad W n_{3}=0.025$ characteristics of the spectral performance function are illustrated in Fig. 6.

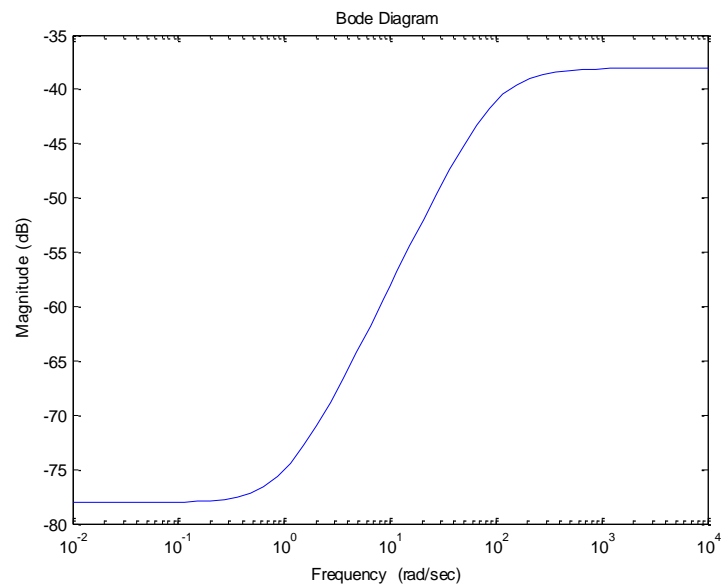

Figure 6

Amplitude frequency characteristics of the shaping filter

Required quality of controlling an aircraft with a control handle and pedals is achieved only when the difference between the required and actual value $s$ is minimized, which is a feedback signal. The $W p$ filter shaper, which narrows the noise band down to $5 \%$ difference with transfer function (Fig. 7, Fig. 8), Zero/Pole placement of the filters $(12,13)$ shows normal displacement in the negative part of the complex plane with conjugated complex roots with negative real parts:

$$
W_{p}=\frac{0.05 s^{4}+2.9 s^{3}+105.9 s^{2}+6.17 s+0.16}{s^{4}+9.19 s^{3}+30.8 s^{2}+18.83 s+3.95}
$$




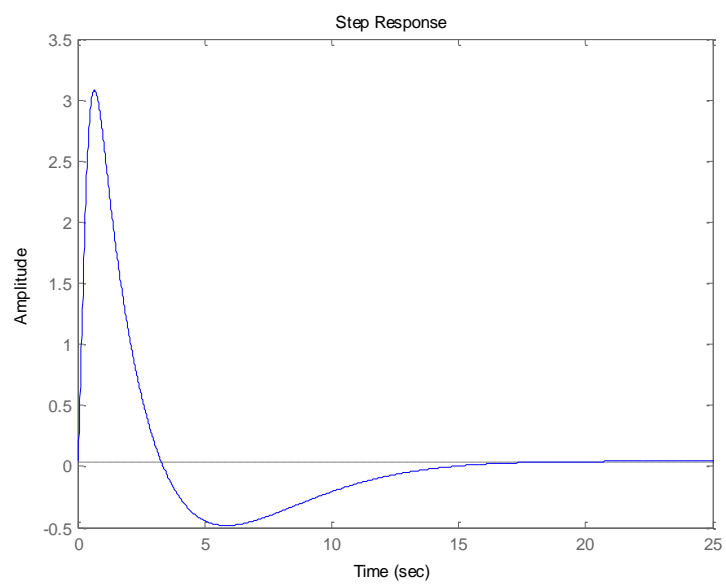

Figure 7

Step response of the Wp differential filter

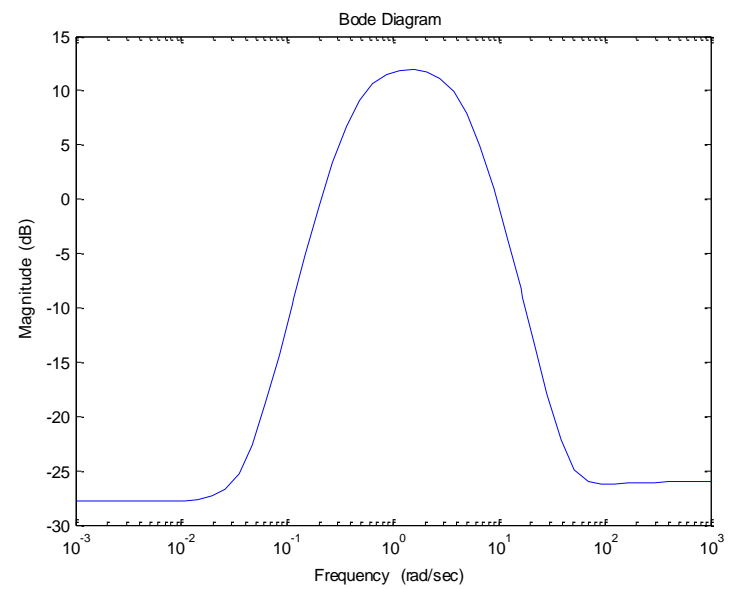

Figure 8

Amplitude frequency characteristics of the Wp differential filter

Directional control of aircraft is of higher quality, which is estimated by the multiple of 2, by which the filtration band is extended (Fig. 9). Then: $W \beta=2 \cdot W p$.

$$
W_{\beta}=\frac{0.1 s^{4}+5.8 s^{3}+211.9 s^{2}+12.34 s+0.32}{s^{4}+9.19 s^{3}+30.8 s^{2}+18.83 s+3.95}
$$




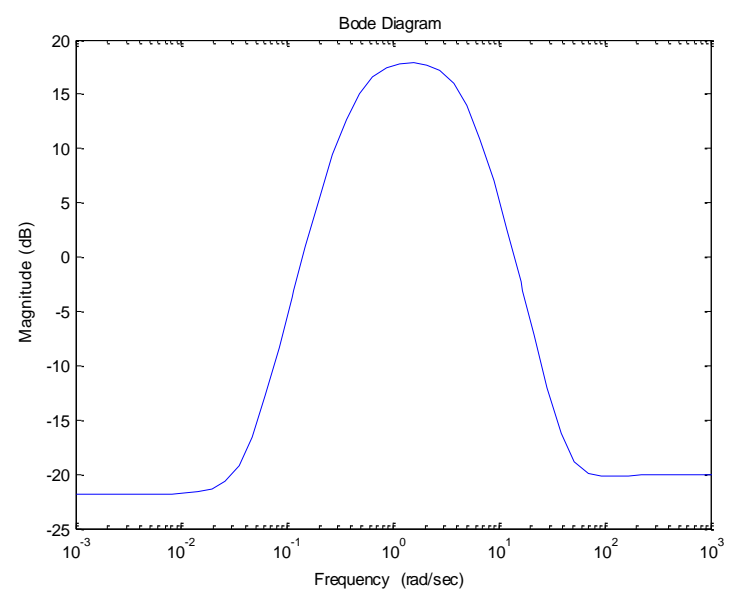

Figure 9

Amplitude frequency characteristics of the $W \beta$ differential filter

Weight functions of Wact, $W n, W p, W \beta$ are closing the circuit between external inputs and weight functions.

\subsection{State Model of an Aircraft in Lateral Motion}

Pilot exercises control of the aircraft by means of control stick handle and pedals, when the controlled inputs are: differential spoilers of lift (interceptors) marked as differential stabilizer deflection $\delta_{\text {int }}[$ deg rees $]$, rudder deflection marked as $\delta_{\text {rud }}[$ deg rees $]$.

The three outputs measured are: roll rate $p$ (deg rees per second), yaw rate $r$ (deg rees per second), lateral acceleration $y_{a c}(g)$ and computed outputs: sideslip angle $\beta$, bank angle $\phi($ deg rees $)$.

State variables are contained in the relation:

$\dot{\boldsymbol{x}}(t)=\boldsymbol{A} \boldsymbol{x}(t)+\boldsymbol{B} \boldsymbol{u}(t)$,

$\boldsymbol{y}(t)=\boldsymbol{C x}(t)$.

where, for the nominal model of the maneuvering aircraft (numerical values taken from [7]) can be set as: 


$$
\begin{aligned}
& \left(\begin{array}{c}
\dot{v} \\
\dot{r} \\
\dot{p} \\
\dot{\phi} \\
\hline \beta \\
p \\
r \\
y_{a c}
\end{array}\right)=\left[\begin{array}{c|c}
A & B \\
\hline C & D
\end{array}\right]\left[\begin{array}{c}
v \\
r \\
p \\
\phi \\
\delta_{\text {differ }} \\
\delta_{\text {rud. }}
\end{array}\right] \\
& {\left[\begin{array}{l|l}
A & B \\
\hline C & D \\
\hline
\end{array}\right]=} \\
& {\left[\begin{array}{cccc|cc}
-0.116 & -227.3 & 43.02 & 31.63 & 0.062 & 0.101 \\
0.003 & -0.26 & -0.14 & 0 & -0.005 & -0.011 \\
-0.021 & 0.67 & -1.37 & 0 & -0.047 & 0.004 \\
0 & 0.19 & 1 & 0 & 0 & 0 \\
\hline 0.247 & 0 & 0 & 0 & 0 & 0 \\
0 & 0 & 57.3 & 0 & 0 & 0 \\
0 & 57.3 & 0 & 0 & 0 & 0 \\
-0.003 & -0.008 & 0.05 & 0 & 0.0029 & 0.002
\end{array}\right]}
\end{aligned}
$$

For lateral motion of the aircraft as a response to the aileron deflections, we get the following transfer functions including actuator dynamics models and are simulated in Figure 10 [7]:

$$
\begin{aligned}
\frac{v(s)}{\xi(s)} & =\frac{0.01536 s^{3}-0.1759 s^{2}-0.1541 s-0.006312}{s^{4}+1.74 s^{3}+2.151 s^{2}+1.762 s+0.004} \\
\frac{r(s)}{\xi(s)} & =\frac{-2.674 s^{3}-1.28 s^{2}-3.173 s+0.004062}{s^{4}+1.74 s^{3}+2.151 s^{2}+1.762 s+0.004} \\
\frac{p(s)}{\xi(s)} & =\frac{-0.3009 s^{3}-0.0491 s^{2}-0.5575 s-0.02192}{s^{4}+1.74 s^{3}+2.151 s^{2}+1.762 s+0.004} \\
\frac{\phi(s)}{\xi(s)} & =\frac{0.0028 s^{4}+0.0025 s^{3}+0.00708 s^{2}+0.00409 s+0.00009}{s^{4}+1.74 s^{3}+2.151 s^{2}+1.762 s+0.004}
\end{aligned}
$$



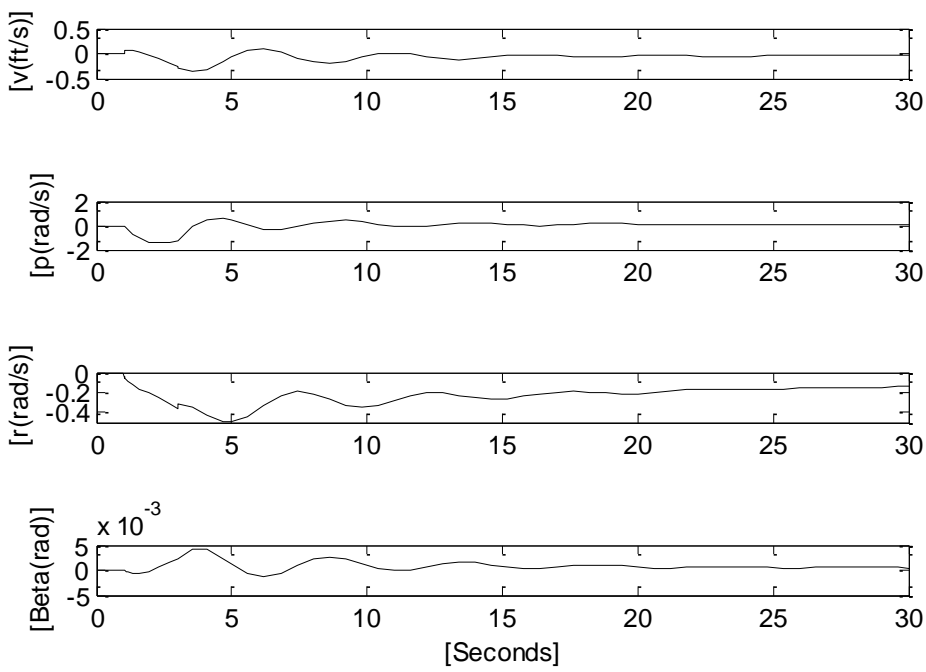

Figure 10

Nominal aircraft response to $1^{\circ} 2 \mathrm{~s}$ aileron pulse input

Lateral motion of aircraft as a response to the rudder deflection is described by the following transfer functions including actuator dynamics models and are simulated in Figure 11 [7]:

$\frac{v(s)}{\zeta(s)}=\frac{0.025 s^{3}+0.708 s^{2}+0.830 s+0.0038}{s^{4}+1.74 s^{3}+2.151 s^{2}+1.762 s+0.004}$

$\frac{r(s)}{\zeta(s)}=\frac{0.208 s^{3}-0.475 s^{2}-3.026 s+0.0039}{s^{4}+1.74 s^{3}+2.151 s^{2}+1.762 s+0.004}$

$\frac{p(s)}{\zeta(s)}=\frac{-0.64 s^{3}-0.9661 s^{2}-0.626 s-0.212}{s^{4}+1.74 s^{3}+2.151 s^{2}+1.762 s+0.004}$

$\frac{\phi(s)}{\zeta(s)}=\frac{0.002 s^{4}+0.003 s^{3}-0.0035 s^{2}-0.008 s+0.00005}{s^{4}+1.74 s^{3}+2.151 s^{2}+1.762 s+0.004}$ 

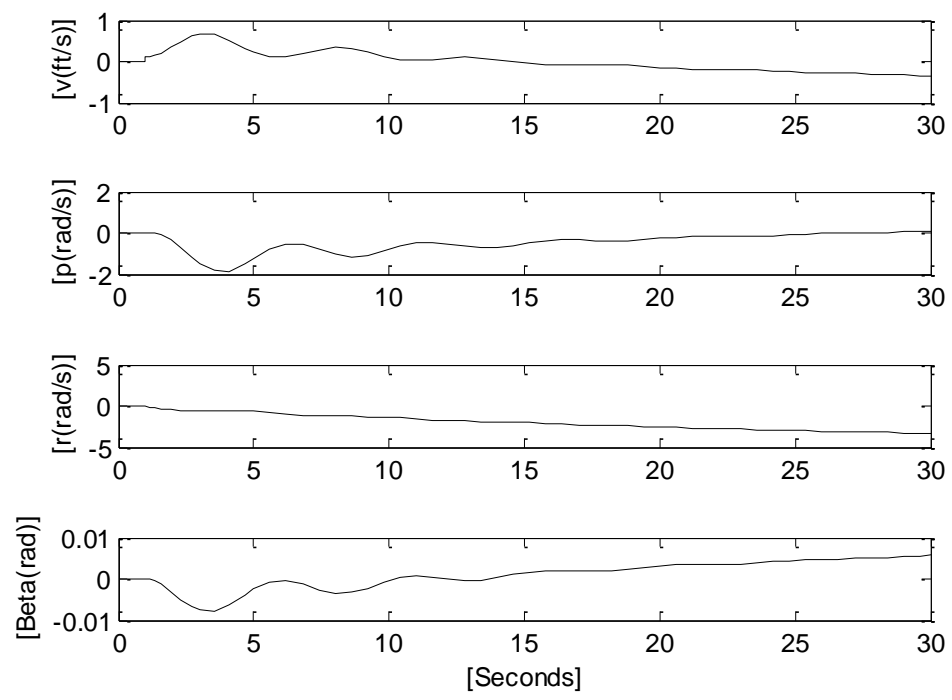

Figure 11

Nominal aircraft response to $1^{\circ}$ rudder step input

The given model is further expanded by actuators $A_{i n t}, A_{\text {rud }}$ with their respective transfer functions modeling system's dynamics with two transfer functions that are identical for rudder and interceptor:

$$
A_{\text {int }}=\frac{25 s}{s+25}, A_{\text {int }_{2}}=\frac{25}{s+25}, A_{\text {int }}=A_{\text {rud }}
$$

Step responses of actuators representing the servo system of the rudder and interceptor drive are shown in Figure 12. 


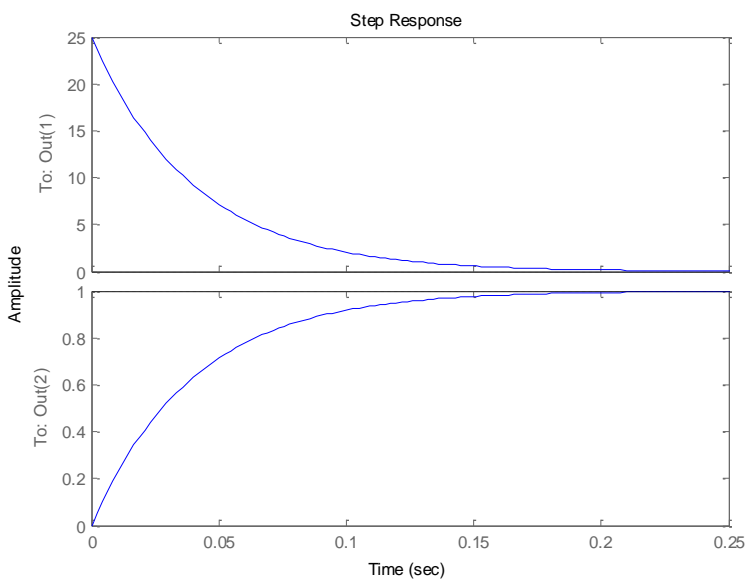

Figure 12

Step response of the actuators of the tail unit

\subsection{Simulation Model Including Errors}

Nominal model of the maneuvering aircraft is approximation from its real characteristics. Errors resulting from the difference are perceived by us as insensitivity, which is modeled by the product of aircraft uncertainties $W_{n e}$ and the first frequency $\Delta G$, which is the output of the weight filter: $W_{n e} \sqsubset G$.

The highest frequency of the weight filter $W_{n}$ is carrying the uncertainty in aircraft control. Frequencies, which differentiate the model from the frequency of the signal of flight dynamics are generated by filters with transfer functions: $W_{1}=\frac{2 s+8}{s+160}, W_{2}=\frac{1.5 s+30}{s+200},[9,11]$. Error of the maneuvering aircraft model is expressed by frequency as in Fig. 13. 


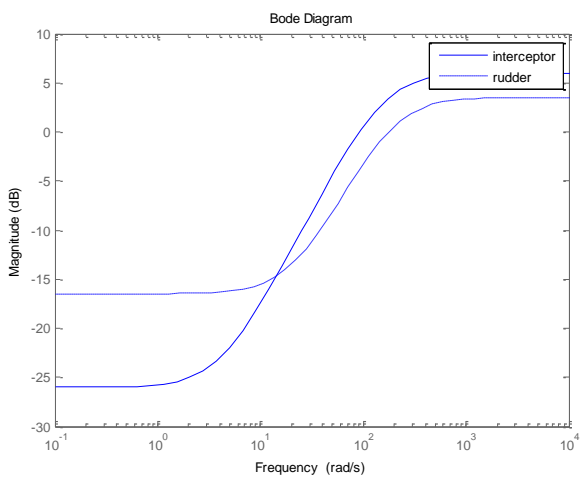

Figure 13

Error of the model of maneuvering aircraft dependant on flight dynamics expressed in frequency characteristics for rudder and interceptor

Weight filters $W_{n e}, \Delta G$ along with the acuators $A_{\text {int }}, A_{\text {rud }}$ are bringing the simulation model closer to real aircraft characteristics.

\subsection{Synthetic Model of a Maneuvering Aircraft}

The synthetic model is formed by a complex scheme of filter transfer functions and actuators in MATLAB environemnt executed with "sysic" command. The main object of this analysis is dynamic behavior of the synthetic aircraft model with influence of errors on outer parameters of stick/pedal control shaping the $\Delta G$. The signal has been processed by application of the MONTE CARLO method. As an example, the differential interceptor circuit was used with the uncertainty of weight $W_{1}$ with $5 \%$ error, where the $100 \%$ error corresponds to angular speed of $93 \mathrm{rad} / \mathrm{sec}$. The analysis is realized in the frequency area with application of Bode method and is done with 10 different noise signals which were selected from the performance spectrum of noises. Results representing all 10 different noise signals are shown in Figure 14. Figure 15 shows step response and frequency analysis concentrating on a single noise. 


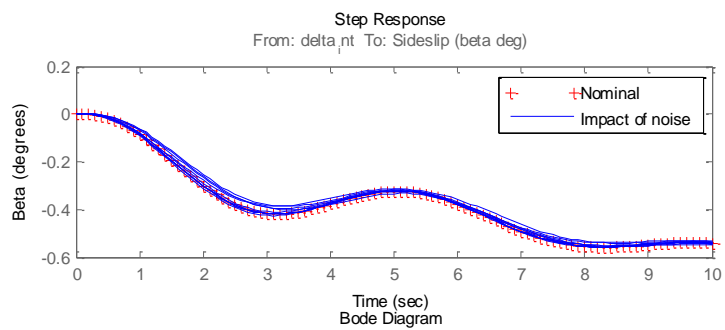

From: delta nt To: Sideslip (beta deg)

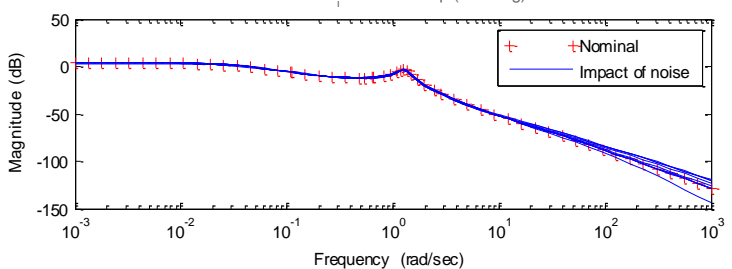

Figure 14

Analysis of 10 different applied noise samples in time domain (step response) and frequency domain

(Bode plot)
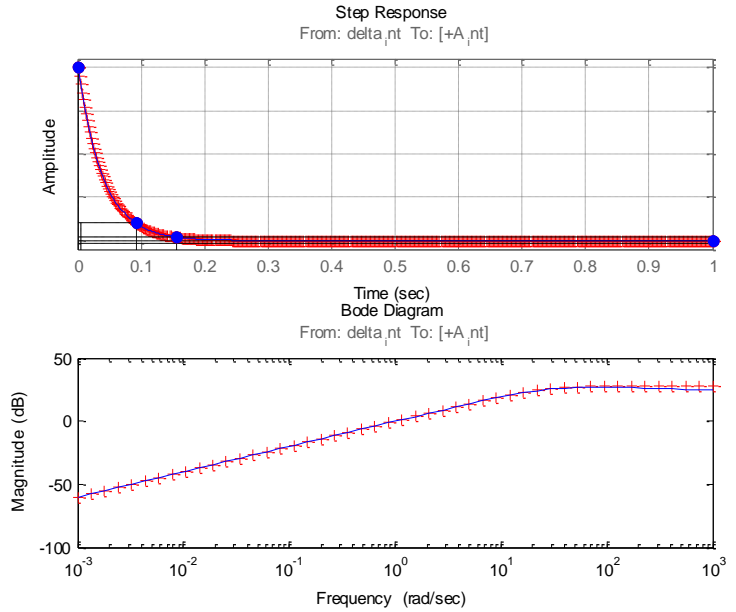

Figure 15

Analysis of the first noise sample in the time and frequency domain selected from the graph of the performance spectrum of noises of the differential interceptor circuit 


\subsection{Simulation Synthesis of the Robust Controller and the Lateral Control of Aircraft}

The design is aimed at such a kind of robust controller, which will be sensitive to the errors being higher in value than the width of insensitiveness $W_{n e}$. In this process the 'sysic' command is used, which enables solution of the problem assuming higher number of outputs than inputs. The approximate status model of the controller generates the F14IC command, [6]. The closed feedback is completing the ADAD, which increases the quality of control. Further steps minimize the closed circuit of the $H_{\infty}$ controller for controlling the nominal model of the maneuvering aircraft with the number of measurement sites at 5 and two control circuits. Using the 'hinfsyn' command helps calculate the controller, and on the command of 'kinf' the controller is adjusting the gain of the feedback. The size of the signal is somewhere between $0.67<1$, in accordance with the model dynamics. By the method of $\mu$ - synthesis, we determine the robust circuit, through which the modeled errors or $\square G$ uncertainty are lead. Using the 'dksyn' command, the mentioned synthesis is performed; the width of the frequency band can be illustrated by using the 'dkitopts' command.

In this case, we compare the $H_{\infty}$ robust controller and with the use of frequencies of feedback signals, their gain is set in a way to conform to each feedback.

By analyzing the sample of the frequency response from the band of feedback uncertainty, the uncertainty of the frequency response is created, [5]. The following figure (Fig. 16) shows obtained gains by both methods comparing the nominal plant and worst case scenario. 

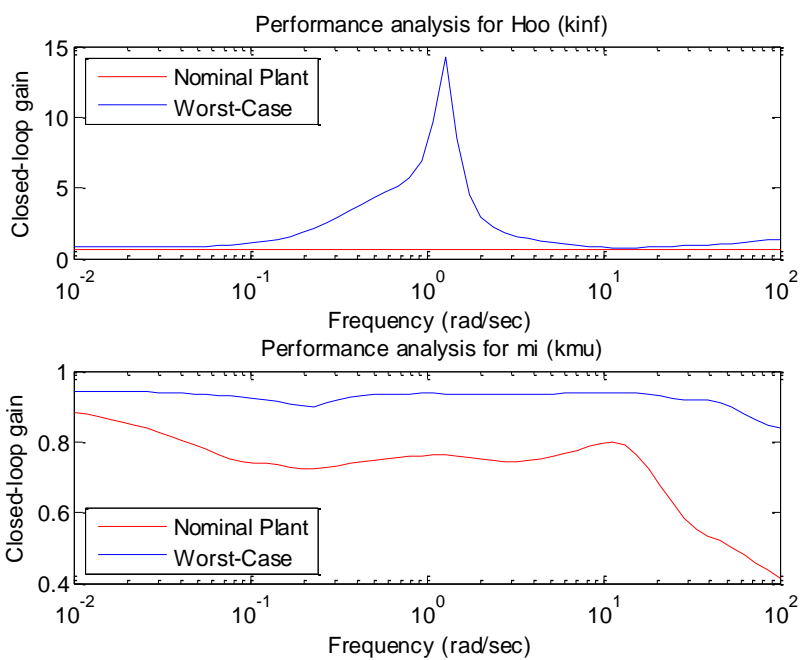

Figure 16

Comparing frequencies for amplification of the feedback for the designed types of controllers

\subsection{Time Domains Validation of the Robust Controller}

The test of the $\mu$ controller in time domain is done by comparison of its response on typical signal (Fig. 17). Three different models were tested - an ideal model, a nominal model with actuators, and disturbed model with actuators and disturbed dynamics by noise.
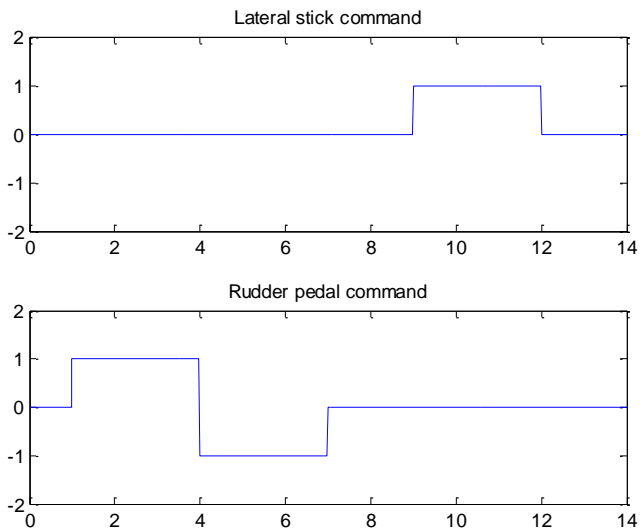

Figure 17

Input type signal for lateral control of aircraft 


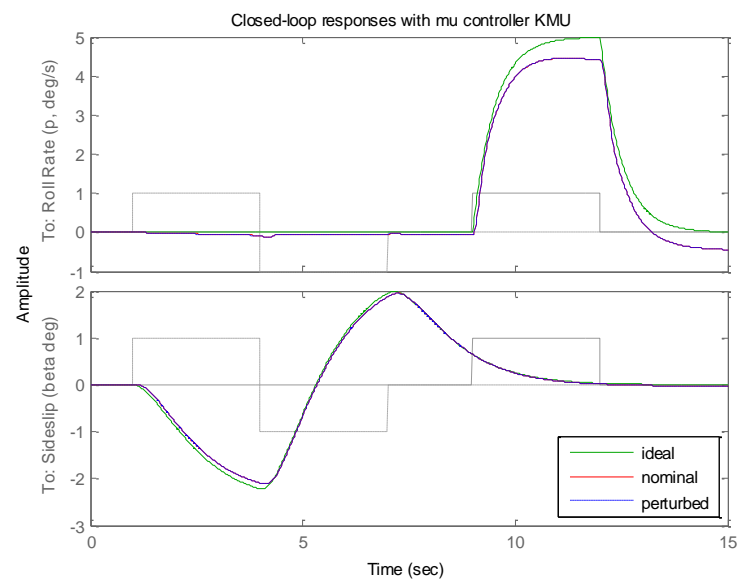

Figure 18

Comparing the response to the control action of the pilot from the closed circuit with the designed controller

Noise frequency was selected that produces worst aircraft behavior was selected and tested. As it is shown in Figure 18, the disturbed model with designed robust controller shows appropriate behavior with error not exceeding the value of 0.024 $\mathrm{rad} / \mathrm{sec}$.

\section{Conclusions}

ADAD systems, which are damping relaxation oscillations, are used to attenuate oscillation of aircraft in the circuit of rudder, where current aircraft, especially at medium altitudes are known for insufficient damping. When flying at large angles of attack, in landing mode, the effect is very small. It results from aerodynamic laws that the oscillation of aircraft in heading generates oscillations in the circuit of pitching, which is known for very high amplitudes. The value of the artificial attenuation constant introduced by the damper into the rudder circuit must be higher. Increase in the attenuation in the rudder circuit is positively reflected in the pitching circuit of the aircraft. Increasing the value of proportional damping of relaxation frequencies brings positive effects for aircraft flying in a turbulent atmosphere, in which the attenuator ensures „lower sensibility" to wind. Apart from improving dynamic features of the aircraft, the ADAD system reduces the stabilized value of the angular velocity when deflecting the control stick by a single degree. Methods of analysis and synthesis helped us determine the parameters of the robust controller of the ADAD system for lateral control of high-maneuverability aircraft. The suggested $H_{\infty}$ controllers shows the example of degradation in the frequency response of flight conditions of the model for the lateral movement of aircraft, when the value of gain is close to 15 . By contrast, the 
$\mu$ controller degrades flight characteristics, when compared to the $H_{\infty}$ controller, as the gain of the feedback is only slightly exceeding the value of 1.25 , consequently, the $\mu$ controller is better in compensating for control errors, if committed.

\section{Acknowledgement}

The work presented in this paper was supported by VEGA, Grant Agency of Ministry of Education and Academy of Science of Slovak Republic under Grant No. 1/0298/12 - "Digital control of complex systems with two degrees of freedom" and grant No. 1/1117/11 - "Integration of automatic flight control algorithms with control algorithms of aircraft turbocompressor engines". The work presented in this paper was also supported by KEGA under Grant No. 018TUKE4/2012 - "Progressive methods of education in the area of control and modeling of complex systems object oriented on aircraft turbo-compressor engines.

\section{References}

[1] ADAMS, Richard J. - BUFFINGTON, James M. - SPARKS, Andrew G. - BANDA, Siva S.: Robust Multivariable Flight Control. London: Springer-Verlag, 1994, ISBN 3-540-19906-3

[2] SONNEVELDT, L.: Nonlinear F-16 Model Description - Version 1.0: Delft:Control \& Simulation Division, Faculty of Aerospace Engineering, Delft University of Technology, March 2010

[3] MACKENROTH, Uwe: Robust Control Systems. Berlin: Springer-Verlag, 2004, ISBN 3-540-20929-9-8

[4] SALIMBAHRAMI, Behnam: Multivariable Robust Control. Lehrstuhl für Regelungstechnik Technische Universität München, 2007

[5] SZÁSZI, István - KULCSÁR, Balázs: Robust Control and Fault Detection Filter Design for Aircraft Pitch Axis. Periodica Polytechnica, roč. 29, č. 1-2 (2001), s. 83-100

[6] Control of F-14 Lateral Axis Using mu-Synthesis. Matlab R2010b Help

[7] BALAS, Gary - CHIANG, Richard - PACKARD, Andy - SAFONOV, Michael: Robust Control Toolbox ${ }^{\mathrm{TM}} 3$ - User's Guide. The MathWorks, Inc., April 2011

[8] ROSKAM, Jan: Airplane Flight Dynamics and Automatic Flight Controls Part I, Third Printing. Lawrence, Kansas: DARcorporation, 2001, ISBN 1884885-17-9

[9] ROHÁČ, J.; REINŠTEIN, M.; DRAXLER, K.: Data Processing of Inertial Sensors in Strong-Vibration Environment. In Intelligent Data Acquisition and Advanced Computing Systems (IDAACS). Piscataway: IEEE, 2011, Vol. 1, pp. 71-75, ISBN 978-1-4577-1426-9 
[10] SOTÁK, Miloš - SOPATA, Milan - BRÉDA, Róbert - ROHÁČ, Jan VÁCI, Luboš: Integrácia navigačných systémov. Monografia. Košice 2006, str. 64 - 65. ISBN 80-969619-9-3

[11] SOTAK, M.: Determining Stochastic Parameters Using an Unified Method. In: Acta Electrotechnica et Informatica. - ISSN 1335-8243, Vol. 9, No. 2 (2009)

[12] LAZAR, T., PASTOR, P.: Factors Limiting Controlling of an Inverted Pendulum. Acta Polytechnica Hungarica Vol. 8, No. 4, (2011), s 23-34

[13] VAŠČÁK, J., MADARÁSZ, L.: Automatic Adaptation of Fuzy Controllers. Acta Polytechnica Hungarica Vol. 2, No. 2, (2005), s 5-18

[14] DURHAM, W. C.: Aircraft Dynamics. Virginia: Virginia Polytechnic Institute \&State University, 2002

[15] Richard J. ADAMS, James M. BUFFINKTON, ANDREW G. SPARKS and Siva S. BANDA: ROBUST MULTIVARIABLE FLIGHT CONTROL. Springer - Verlag, Berlin Heidelbeberg New Yourk. ISBN 3-540-19906-3

[16] R. P. G. Collinson: Introduction to Avionics. ISBN 0412-48250-9 